\title{
NOVA GK PERSEI - A MINIATURE SUPERNOVA REMNANT?
}

\author{
E. R. Seaquist and D. A. Frail, University of Toronto, Canada \\ M. F. Bode, J. A. Roberts, and D. C. B. Whittet, Lancashire Polytechnic, U. K. \\ A. Evans and J. S. Albinson, University of Keele, U. K.
}

\begin{abstract}
We present radio and optical images of the shell-like remnant of the 1901 outburst of Nova GK Persei. The behaviour of this object is remarkably similar to supernova remnants. The synchrotron radiation-emitting shell is polarized with the magnetic field oriented radially, as in young SNR's. This similarity plus extensive data we have acquired on the expansion and the interstellar environment of GK Per indicate that the nova shell is colliding with ambient gas whose density is substantially higher than the ISM.

Furthermore, there is strong evidence that the ambient gas is circumstellar rather than interstellar, and that this material is the shell of an ancient planetary nebula associated with the white dwarf companion of GK Per.
\end{abstract}

The production of synchrotron radiation-emitting electrons in a supernova remnant (SNR) occurs when the ejecta shell sweeps up interstellar or circumstellar gas with mass comparable to that in the shell. This effect is evidently occurring in the nova remnant GK Persei 1901, as originally discovered by Reynolds and Chevalier (1984).

We have made extensive observations of the nonthermal radio emission from GK Per using the VLA in D configuration at $6 \mathrm{~cm}$ and $\mathrm{C}$ configuration at $20 \mathrm{~cm}$. Figure (1) shows the $6 \mathrm{~cm}$ map superposed on a [NII] CCD image kindly provided to us by H.W. Duerbeck. The bright [NII] emission, together with the coincident radio emission, suggests an interaction between a shell of expanding nova ejecta and an ambient medium located to the southwest. Note, however, that there is no detailed correspondence between radio emission and optical knots. The phenomenon is similar to that occuring in SNR's.

Figure (2) shows the radio spectrum of the integrated emission from GK Per demonstrating that the mechanism is synchrotron radiation, and that a flattening occurs in the spectrum at low frequencies. Our studies show that this flattening must be caused by curvature in the energy distribution of the emitting relativistic electrons, rather than by any absorption process or the Razin Effect.

Figure (3) shows the distribution of polarization E-vectors superimposed on the total intensity contours at $6 \mathrm{~cm}$ and $20 \mathrm{~cm}$, as well as the corresponding distributions of the percentage polarization. The key features are first that the E-vectors at $6 \mathrm{~cm}$ are tangent to the shell signifying a magnetic field directed radially. The $20-\mathrm{cm}$ E-vectors are rotated by galactic Faraday rotation occurring over the intervening $500 \mathrm{pc}$. These characteristics are again reminiscent of a young SNR such as Cas A, except that the entire phenomenon is scaled down by five orders of magnitude in energy!

Note that the $20-\mathrm{cm}$ emission is depolarized relative to that at $6 \mathrm{~cm}$ by a factor of three in the outer part of the intense ridge. This effect is produced by Faraday effects occurring within the ridge itself due to the magnetic field (equipartition value $7 \times 10^{-5}$ gauss) and a plasma with mean electron density of $10-100 \mathrm{~cm}^{-3}$. This plasma probably originates from the [NII]-emitting ejecta, since the depolarization occurs where the [NII] emission is generally the brightest. 


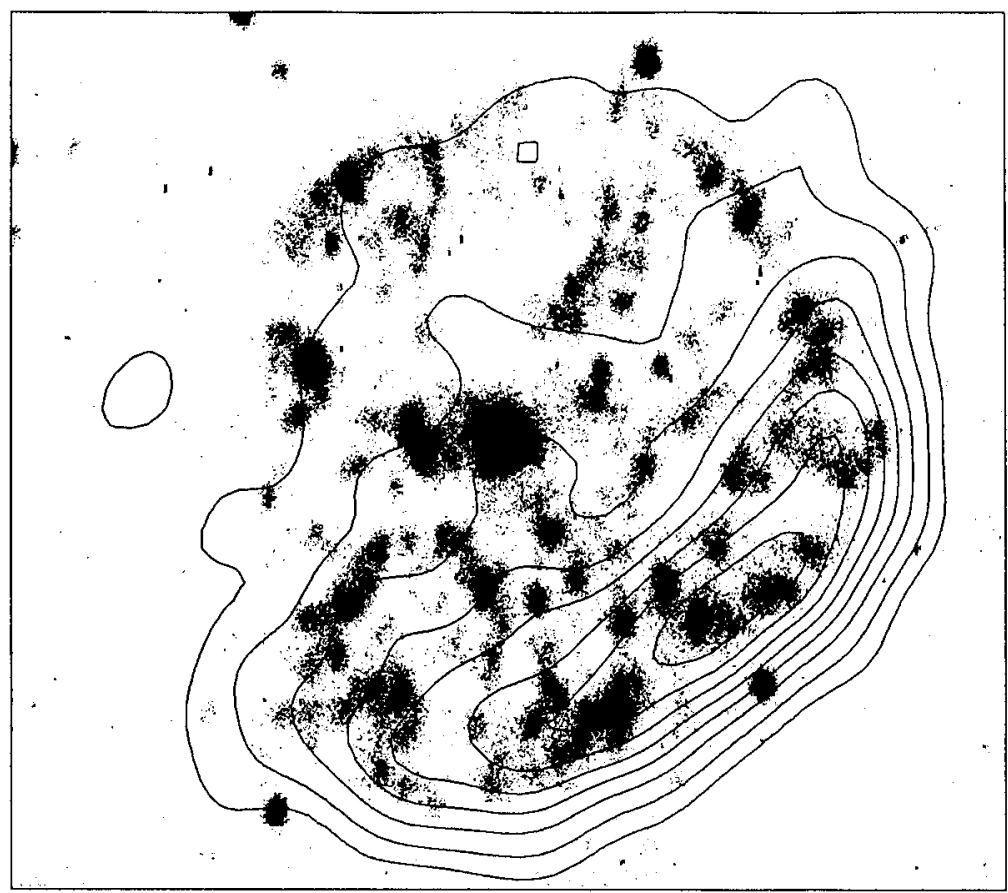

Figure (1) : Radio emission at $6 \mathrm{~cm}$ superimposed on an [NII] CCD image.

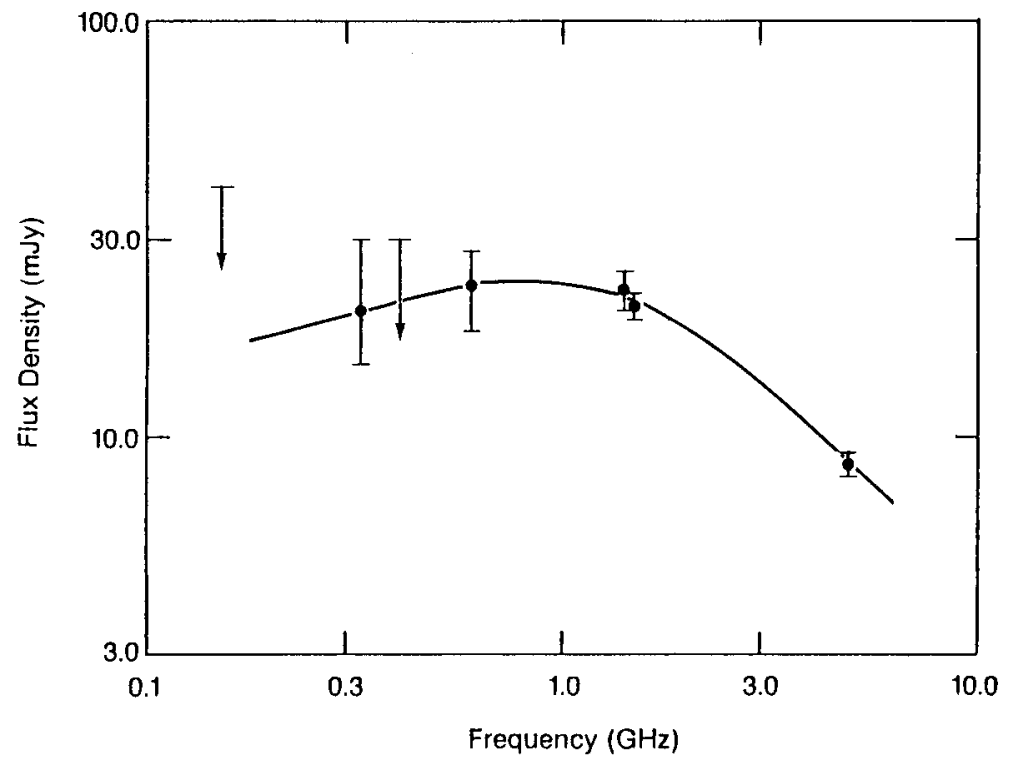

Figure (2) : Integrated radio spectrum of GK Per. 

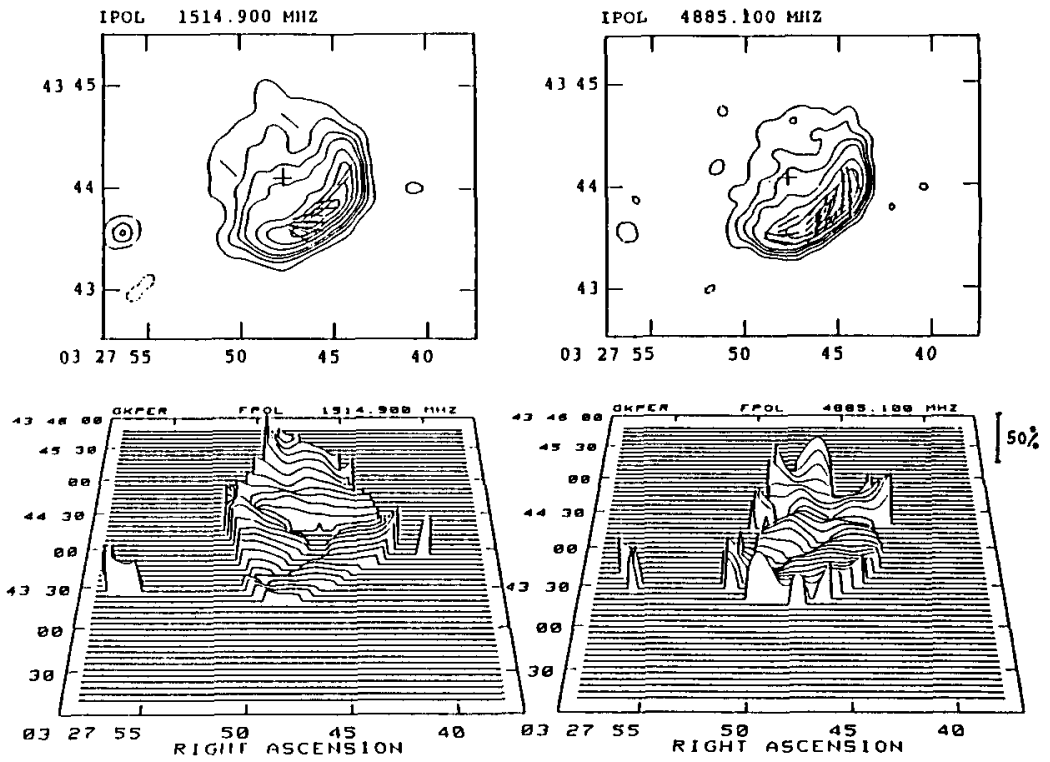

Figure (3) - Top : Polarization vectors at $20 \mathrm{~cm}$ and $6 \mathrm{~cm}$ superimposed on total intensity contours. Bottom : Percentage polarization at $20 \mathrm{~cm}$ and $6 \mathrm{~cm}$.
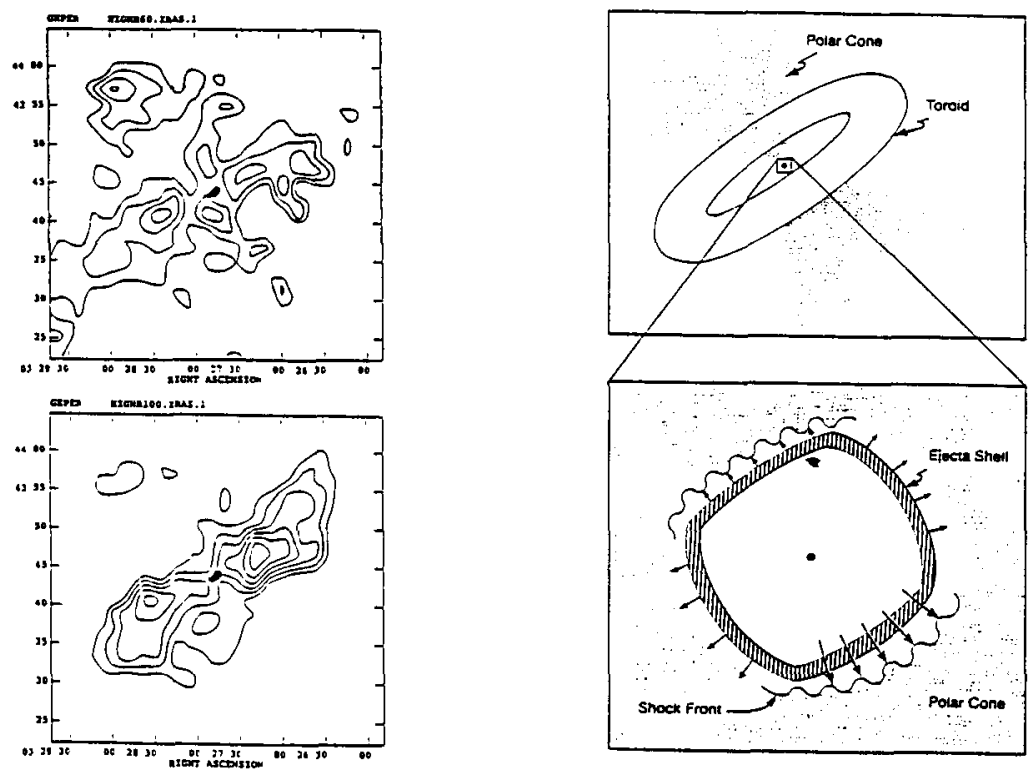

Figure (4) - Left : An IRAS $60 \mu \mathrm{m}$ map (top) and a $100 \mu \mathrm{m}$ map (bottom), showing the IR emission surrounding GK Per. The radio remnant is shown to scale by the blackened spot at the center.

Figure (5) - Right: A schematic showing our interpretation, which involves the interaction between the nova ejecta and the remnant of an ancient planetary nebula associated with GK Per. 
Figure (4) shows IRAS maps at $60 \mu \mathrm{m}$ and $100 \mu \mathrm{m}$ of an extended region surrounding GK Per. The position of the GK Per is shown to scale as the black dot in the middle of the IR contours. Evidently GK Per is at the centre of a cloud of dust with maximum dimension $4 \mathrm{pc}$ and with GK Per situated in a saddle of the IR brightness distribution. The cloud appears elongated in the same direction as the radio emitting ridge of the remnant. The colour temperature of the dust is about $23 \mathrm{~K}$, similar to that of the IR cirrus. HI 21-cm emission from this region (not shown) suggests an elongated feature parallel to the axis of the IR emission. Analyses of these data suggest that GK Per is embedded in a toroidal ring of gas and dust whose radius and total mass are respectively about $1 \mathrm{pc}$ and $1 \mathrm{M}_{\odot}$, and that gas associated with this cloud is responsible for the interaction which produces the synchrotron radiation.

We are led to hypothesize that the cloud surrounding GK Per is an ancient planetary nebula, associated with the evolution of one member of the binary to the white dwarf stage (Bode et al. 1987a). The morphology of the nebula is shaped by the presence of the binary. The age of this planetary would be between 50,000 and 200,000 years. Scattering of light by the dust associated with the planetary nebula may have been responsible for the apparent "superluminal" expansion of the nebulosity in this nova seen at the time of the 1901 outburst (Couderc 1939). Figure (5) shows how the nova ejecta may be interacting with the remnant of a bipolar outflow associated with the planetary nebula phase whose axis is coincident with that of the torus. The association of such bipolar structures with planetary nebulae is fairly common (Balick 1987). If this picture is correct, then the observed interaction would be possible only if the 1901 nova outburst in this system is the first. Otherwise the medium interacting with the ejecta would have been swept away by a previous outburst.

A search for nonthermal emission from other old fast novae similar to GK Per failed to turn up similar phenomena in other cases (Bode 1987b). Thus the phenomenon appears so far to be unique to GK Per.

\section{Conclusion:}

The radio and optical features associated with GK Per, coupled with the morphology of extended IR emission surrounding the nova, suggest to us that the nova ejecta are presently interacting with an ancient planetary nebula associated with GK Per. A detailed study shows that the interaction is very similar to that produced in young SNR's. The uniqueness of this phenomenon among novae supports the case for an interaction of a nova with its own pranetary nebula.

If this picture is correct, then the study of this object may provide some unusual insights into relativistic particle production in shock waves, and into the timescales involved in the production of white dwarfs and in the onset of nova outbursts.

\section{Acknowledgements:}

ERS acknowledges the support of an operating grant from the National Sciences and Engineering Research Council of Canada (NSERC). DAF was supported by an NSERC scholarship. MFB and JSA are supported by the UK Science and Engineering Research Council. JAR is supported by the by the National Advisory Body to the Polytechnics.

\section{References:}

Balick, B., in Proceedings of the Calgary Workshop on Late Stages of Stellar Evolution, Calgary, Alberta, Canada, June $\mathscr{2}-5,1986$, in press, 1987.

Bode, M. F., Seaquist, E. R., Frail, D. A., Roberts, J. A., Whittet, D. C. B., Evans, A., and Albinson, J. S., submitted to Nature, 1987a.

Bode, M. F., et al., Mon. Not. R. Astron. Soc., in press, 1987b.

Couderc, P., Ann. d'Astrophys., 2, 271, 1939.

Reynolds, S. P. and Chevalier, R. A., Ap. J. (Letters), 281, L33, 1984. 